\title{
TOPOLOGICAL DIVISORS OF ZERO AND TAUBERIAN THEOREMS
}

BY

\section{ALBERT WILANSKY}

1. Introduction. The connection between the two topics mentioned in the title has been shown by I. D. Berg [2]. (See Theorem 6, below.) The author thanks Dr. Berg for assistance with the material of this article.

The topological divisors of 0 in the algebra $B[X]$ of all bounded endomorphisms of a Banach space $X$ are fairly well understood $[15 ; 22]$. In this article we shall extend this knowledge to a subalgebra of $B[X]$, and deduce consequences in the form of Tauberian theorems.

Algebraists will find no very deep algebraic results in the extension, but they may be interested to see how algebraic properties correspond to analytic concepts.

The very oldest such correspondence is undoubtedly the remark that if a transformation is invertible in the algebraic sense, it is trivial in the summability sense in that it carries no divergent sequences into convergent ones.

2. Notation. All the notation given, except for $\psi$ and $\chi_{n}$, is standard, and may be found, for example, in $[14 ; 15 ; 18 ; 19]$.

By $c_{0}, c, m$ are meant, respectively, the spaces of null, convergent and bounded sequences $x=\left\{x_{n}\right\}, n=1,2, \cdots$. For $x \in c_{0}, c, m,\|x\|=\sup \left|x_{n}\right|$. The space of sequences $x$ with $\Sigma\left|x_{n}\right|<\infty$ is written $l$. The constant sequence of ones is written 1 , and $\delta^{n}, n=1,2, \cdots$, is the sequence whose $n$th term is 1 , all other terms 0 .

If $A=\left(a_{n k}\right), n, k=1,2, \cdots$, is a matrix, $A x$ is defined to be $\left\{(A x)_{n}\right\}$ where $(A x)_{n}=\sum_{k=1}^{\infty} a_{n k} x_{k}$, and $x$ is called summable by $A$ if $x \in c_{A}$ where $c_{A}=\{x: A x \in c\}$, and $\lim A x$ is also written $\lim _{A} x$.

If $c_{A} \supset c, A$ is called conservative. If $\lim _{A} x=\lim x$ for all $x \in c, A$ is called permanent. (We avoid the more usual term "regular" which has a quite different significance in algebra. For the same reason we deplore the use of the word "normal" to describe the shape of certain matrices.)

If $\lim _{A} x=t \lim x$ for all $x \in c, A$ is called multiplicative-t.

$B[X]$ stands for the Banach algebra of endomorphisms (always assumed bounded) of any Banach space $X$, with $\|T\|=\sup \{\|T x\|:\|x\| \leqq 1\}$, and $X^{\prime}$ is the space of continuous linear functionals on $X$.

An endomorphism which is one-to-one and onto is called an automorphism.

Presented to the Society, April 30, 1963, under the title Divisors of zero and Tauberian theorems; received by the editors March, 1963 and, in revised form, June 6, 1963. 
If $T \in B[X]$, and $T[X]$ is closed, $T$ is said to be range-closed.

$\Gamma$ will denote the subset of $B[c]$ consisting of those endomorphisms which are given by matrices.

For any matrix $A$, whether conservative or not, we define $\|A\|=\sup _{n} \sum_{k=1}^{\infty}\left|a_{n k}\right|$. In case $A \in \Gamma$, this is equal to its norm as a member of $B[c]$.

If $Y$ is a Banach algebra and $y \in Y$, we call $y$ a left divisor of 0 if there exists $z \in Y$ with $y z=0 \neq z$. Similarly right divisor of 0 and (two-sided) divisor of 0 are defined. $y$ is called a left topological divisor of 0 if for each $\varepsilon>0$, there exists $z$ with $\|z\|=1,\|y z\|<\varepsilon$; again, similar definitions are given with "left" replaced by "right", and "two-sided."

Writers in summability have been accustomed to use the phrase "of type $M$ ", to designate matrices which are not right divisors of 0 in $\Gamma$.

Because of this nonuniformity of terminology, some connections between the subjects have been overlooked. For example, Theorem II of [13] is the special case of Theorem 3.3 of [22] in which $X=Y=c_{0}$.

For $u \in c, f \in c^{\prime}$ we define $u \otimes f \in B[c]$ by $[u \otimes f](x)=f(x) u$.

For $f \in c^{\prime}$, let $\Psi(f)=f(1)-\sum f\left(\delta^{k}\right)$, so that $f(x)=\Psi(f) \lim x+\sum f\left(\delta^{k}\right) x_{k}$ for $x \in c$.

For $T \in B[c]$, let $\chi(T)=\Psi(\lim \circ T)$.

In case $T$ is a matrix this is a familiar definition.

$T$ is called conull if $\chi(T)=0$, otherwise coregular.

For a sequence $x$, let $P_{n} x=x_{n}$, thus defining the functionals $P_{n}$ for $n=1,2, \cdots$. Let $\chi_{n}(T)=\Psi\left(P_{n} \circ T\right)$ for $n=1,2, \cdots$.

3. Algebraic preliminaries. The results of this section are mostly known, or adaptations of known ideas. They are being presented in a form suitable for their later application.

It can now be seen that $T$ is a matrix if and only if $\chi_{n}(T)=0$ for all $n$ (since $\left.P_{n} \circ T x=\chi_{n}(T) \lim x+\sum\left(P_{n} \circ T \delta^{k}\right) x_{k}\right)$. Thus $\Gamma$ is the intersection of a family of closed hyperplanes, hence is closed in $B[c]$.

If $T$ is a matrix we have $T=\left(t_{n k}\right)$ with $t_{n k}=P_{n} \circ T \delta^{k}$ and $\|T\|=\sup _{n} \Sigma_{k}\left|t_{n k}\right|$, as is fairly straightforward to check.

The results collected in Lemma 1 are all obvious.

LEMma 1. Let $T \in B[c], f \in c^{\prime}, u \in c$. Then

(a) If $T^{2}=0, I+T$ is invertible, indeed $(I+T)^{-1}=I-T$.

(b) If $f(u)=0,[u \otimes f]^{2}=0$.

(c) $\chi(u \otimes f)=\Psi(f) \lim u$.

(d) $\chi_{k}(u \otimes f)=\Psi(f) u_{k}$.

(e) $1 \otimes f$ is a matrix if and only if it is conull.

We now conduct some computations involving $\chi$.

LEMMA 2. For $f \in c^{\prime}, T \in B[c]$ we have $\Psi(f \circ T)=\Psi(f) \chi(T)+\sum f\left(\delta^{\prime}\right) \chi_{r}(T)$. 
For $x \in c, f[T x]=\Psi(f) \lim T x+\Sigma f\left(\delta^{r}\right) P_{r} \circ T x$.

Now

and

$$
\lim T x=\Psi(\lim \circ T) \lim x+\sum \alpha_{k} x_{k}
$$

$$
P_{r} \circ T x=\Psi\left(P_{r} \circ T\right) \lim x+\Sigma \beta_{k} x_{k},
$$

where the $\alpha_{k}$ and $\beta_{k}$ are immaterial.

Thus $f[T x]=\left[\Psi(f) \chi(T)+\Sigma f\left(\delta^{r}\right) \chi_{r}(T)\right] \lim x+\sum \gamma_{k} x_{k}$, and the result follows.

Observe the consistent result obtained by taking $f=\lim$ or $f=P_{k}$.

Lemma 3. For $U, V \in B[c]$, we have

$$
\begin{aligned}
& \chi(U V)=\chi(U) \chi(V)+\Sigma\left(\lim \circ U \delta^{r}\right) \chi_{r}(V), \\
& \chi_{k}(U V)=\chi_{k}(U) \chi(V)+\Sigma\left(P_{k} \circ U \delta^{r}\right) \chi_{r}(V), \text { for } k=1,2, \cdots .
\end{aligned}
$$

These formulas follow from Lemma 2 by taking $T=V$, and, respectively, $f=\lim , P_{1}, P_{2}, \cdots$.

Lemma 4. Let $U, V \in B[c]$. Then if $V$ is a matrix, or if $U\left[c_{0}\right] \subset c_{0}$, we have $\chi(U V)=\chi(U) \chi(V)$.

This follows from Lemma 3 since, if $V$ is a matrix, $\chi_{r}(V)=0$ for all $r$, and if $U\left[c_{0}\right] \subset c_{0}, \lim \circ U \delta^{r}=0$ for all $r$.

Lemma 5. Let $U, V \in B[c]$. Then if Vis a matrix, we have $\chi_{k}(U V)=\chi_{k}(U) \chi(V)$.

This follows from Lemma 3 since $\chi_{r}(V)=0$ for all $r$.

LEMMA 6. Let $U, V \in B[c]$. Suppose that Vis a matrix, and $U V$ is a coregular matrix. Then $U$ is a matrix.

It follows from Lemma 4 that $\chi(V) \neq 0$. By hypothesis $\chi_{k}(U V)=0$ for all $k$, and so, by Lemma $5, \chi_{k}(U)=0$ for all $k$. Thus $U$ is a matrix.

Lemma 7. Let $U, V \in B[c]$. Suppose that $V$ is a coregular matrix and that $U V$ is a matrix. Then $U$ is a matrix.

This follows immediately from Lemma 5 .

Lemma 8. Let $X$ be a Banach space and $T \in B[X]$. Then $T$ is a left topological divisor of 0 if and only if to each $\varepsilon>0$ corresponds $x \in X$ with $\|x\|=1,\|T x\|<\varepsilon$.

See, for example, [22, Theorem 3.5].

\section{Algebraic results.}

THEOREM 1. $\Gamma$ contains its inverses in $B[c]$, i.e., if $T \in \Gamma$ and $T^{-1} \in B[c]$, then $T^{-1} \in \Gamma$. Indeed, if $T \in \Gamma$, any left inverse of $T$ also belongs to $\Gamma$. 
This follows from Lemma 6 , taking $U=T^{-1}, V=T$.

The first half of the next result is known [19].

THEOREM 2. The set of conull matrices is an ideal in $\Gamma$, and a left ideal in $B[c]$.

By Lemma $4, \chi$ is a scalar homomorphism on $\Gamma$. Thus $\chi^{\perp} \cap \Gamma$ is an ideal of codimension one in $\Gamma$.

Next, let $U \in B[c]$ and let $V$ be a conull matrix. By Lemmas 4, $5 \chi_{k}(U V)$ $=\chi(U V)=0$ for all $k$, hence $U V$ is a conull matrix.

We remark in passing that $c$ can be embedded in $B[c]$ as a minimal left ideal. Thus, by Theorem 2, each such embedding is either disjoint from, or included in the set of conull matrices. Lemma 1(e) is a special case of this remark.

The arguments used in proving the following result were observed in early articles by R. S. Phillips and A. Sobczyk.

LEMMA 9. Let $S$ be a closed linear subspace of $c$ such that $c, S$ are linearly homeomorphic. Then there exists a bounded projection from $c$ onto $S$.

Let $U: S \rightarrow c$ be the linear homeomorphism. Thinking of $U$ as a map from $S$ into $m$, we may extend it to a bounded linear map $V: c \rightarrow m$. (Merely use the ordinary Hahn-Banach theorem to extend each $P_{k} \circ U$.) Since $V$ is bounded, $V[c]$ is separable. Thus there exists a projection $\pi$ of $V[c]$ onto $c$. (See [10].) Then $U^{-1} \circ \pi \circ V$ is the required projection.

According to [10], the projection just produced has norm $\leqq 3\left\|U^{-1}\right\|\|U\|$, providing, as is always possible, $\|V\|=\|U\|$.

Lemma 9 also gives the usual extension theorems of Hahn-Banach type. The second part of the following result is due to J. Copping [6].

Lemma 10. Let $T \in B[c]$. Suppose that $T$ is one-to-one and $T[c]$ is closed. Then $T$ has a left inverse in $B[c]$, and conversely. If in addition $T \in \Gamma$, then $T$ has a left inverse in $\Gamma$.

Let $U=T^{-1}: T[c] \rightarrow c$. Let $p$ be a bounded projection of $c$ onto $T[c]$ (Lemma 9). Then $U \circ p$ is a left inverse for $T$. (This is the argument of [22, Theorem 3.15].)

THEOREM 3. A conull matrix cannot map c one-to-one and onto a closed subspace of $c$. However, there exists a conull automorphism of $c$.

The first part follows from Lemma 10 and Theorem 2. It also follows from Theorem 5 and Corollary 3, below.

To prove the second part, let $T=I+U$ where $U^{2}=0$ and $\chi(U)=-1$. (See Lemma 1(a).) Such a $U$ is, for example, $1 \otimes\left(P_{1}-\lim \right)$. (See Lemma 1(b)(c).)

In Theorem 3, "one-to-one" cannot be dropped; for example 0 and $1 \otimes P_{1}$ are range-closed conull matrices. See also $\$ 9$, Part I. 
COROLlaRY 1. The isomorphism between $c$ and $c_{0}$ cannot be realized by a matrix map from $c$ onto $c_{0}$.

The standard example is given in $[1, \mathrm{p} .181]$. It is amusing that the isomorphism from $c_{0}$ onto $c$ is given by a matrix.

Theorem 3 shows how the existence of an inverse (Lemma 10) can be applied to obtain a mapping result. This is J. Copping's technique for obtaining Tauberan theorems. (See [6, Theorem 4]. The results are Tauberian rather than Mercerian because of the side condition, boundedness.)

We now turn to what appears to be a more efficient technique, namely the discussion of topological divisors of zero, as suggested by I. D. Berg [2].

We begin with left and left topological divisors of 0 .

Lemma 11. Let $T \in \Gamma$. Then if $T$ is a left divisor of 0 in $B[c]$, it is a left divisor of 0 in $\Gamma$. The same is true with "left" replaced by "left topological."

(In the following proof, we do not use the fact that $T$ is a matrix.)

If $T$ is a left divisor of 0 , say, $T V=0, V \neq 0$. Then $T u=0$ for some $u \neq 0$, $u \in c$, namely $u=V x$ for some $x$, and so $T u \otimes P_{1}=0$.

If $T$ is a left topological divisor of 0 in $B[c]$, either $T$ is a left divisor of 0 in $B[c]$, hence in $\Gamma$, as before, or $T$ is not range-closed [14, pp. 278-279]. Thus, given $\varepsilon>0$, there exists $u \in c$ with $\|u\|>1,\|T u\|<\varepsilon$. For some $n,\left|u_{n}\right|>1$. Let $V=u \otimes P_{n}$. Then $\|V\|>1,\|T V\|<\varepsilon$, and $V \in \Gamma$ by Lemma 1(d).

The study of right divisors of 0 is a very important topic in summability; see for example [1, pp. 90-95] where the nonexistence of a left annihilator is a key assumption. J. D. Hill [8] dubbed a matrix "of type $M$ "' if it is not a right divisor of 0 in $\Gamma$.

The following theorem contains, as a special case, the characterization due to M. S. Ramanujan [13, Theorem II], as well as Lemma 2, p. 93 of [1], and the results of Hanai [7], and Hill [8, Theorem 3].

THEOREM 4. Let $T \in \Gamma$.

(a) Suppose that $T$ is coregular. Then $T$ is of type $M$ (i.e. not a right divisor of 0 in $\Gamma$ ) if and only if the range of $T$ is dense in $c$.

(b) Suppose that $T$ is multiplicative-0. Then $T$ is of type $M$ if and only if the range of $T$ is dense in $c_{0}$.

(c) In all cases, if the range of $T$ is dense in $c, T$ is of type $M$, and if $T$ is of type $M$ the range of $T$ is dense, either in $c$ or in a maximal subspace of $c$.

If $T[c]$ is dense in $c, T$ cannot be a right divisor of 0 . Suppose that $T[c]$ is not dense in $c$. Then there exists $f \in c^{\prime}$ with $f \circ T=0, f \neq 0$. By Lemma 2, $\Psi(f) \chi(T)=0$. (Recall that $\chi_{r}(T)=0$ since $T$ is a matrix.) Thus if $T$ is coregular, $\Psi(f)=0$. Then $\delta^{1} \otimes f$ is a matrix by Lemma $1(\mathrm{~d})$, and $\left(\delta^{1} \otimes f\right) T=0$.

Next, let us assume that the codimension of $\overline{T[c]}$ is at least 2 . There exists a 
nonzero $f \in c^{\prime}$ with $\Psi(f)=0$ and $f(x)=0$ for $x \in T[c]$. (For the subspace of $c^{\prime}$ which vanishes on $T[c]$ is at least two-dimensional.) Thus again $T$ is a right divisor of 0 .

Now $T$ is multiplicative- 0 precisely if $T[c] \subset c_{0}$. If the range of $T$ is not dense in $c_{0}$, its closure has codimension in $c$ at least 2 , and so $T$ is a right divisor of 0 . Conversely, suppose that $\overline{T[c]}=c_{0}$ and $S T=0 \neq S$. For some $k, f=P_{k} \circ S \neq 0$. Since $f=0$ on $c_{0}$, but $f \neq 0$, it follows that $\Psi(f) \neq 0$, i.e., $\chi_{k}(S) \neq 0$ and $S$ is not a matrix. Thus $T$ is not a right divisor of 0 in $\Gamma$.

The ambiguity allowed in Theorem 4 may be realized, i.e., there exist members of $\Gamma$ such that the closure of their range is a maximal subspace, some of which are divisors of zero and some not.

For example $T=\left(t_{n k}\right)$ with $t_{n n}=1, t_{n n-1}=-1, t_{n k}=0$ otherwise, is of type $M$ and its range is included in $c_{0}$. On the other hand, $I-\delta^{1} \otimes P_{1}$ is a projection onto $P_{1}^{\perp}$ and has $\delta^{1} \otimes P_{1}$ as annihilator, so is not of type $M$.

We can say, however, that whether or not $T$ is a right divisor of 0 in $\Gamma$ depends entirely on the closure of its range. Let $\overline{T[c]}$ be a maximal subspace of $c$. Then it may be checked, we omit the proof, that $T$ is of type $M$ if and only if $S \subset m\left(=l^{\prime}\right)$ is total over $l$, where $S$ is the set of all convergent sequences $x=\left\{x_{1}, x_{2}, x_{3}, \cdots\right\}$ such that the sequence $\left\{x_{2}, x_{3}, x_{4}, \cdots\right\}$ belongs to $\overline{T[c]}$. Note that $S$ is a maximal subspace of $c$.

CoRollary 2. If $T$ is coregular, the first result of Lemma 11 is correct with "left" replaced by "right."

This follows from Theorem 4(a), and [22, Theorem 3.3].

As the discussion of Theorem 4 shows, the assumption "coregular" cannot be omitted in Corollary 2.

Finally we consider right topological divisors of 0 . The result of Lemma 11 is false for these. For example, let $T \in \Gamma$ have range not dense, and a right inverse matrix $U$ of finite norm. We might, for instance, take

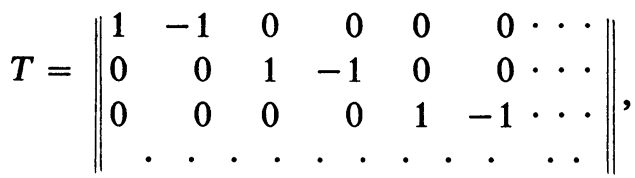

$$
\begin{aligned}
& U=\left\|\begin{array}{llll}
1 & 0 & 0 & \cdots \\
0 & 0 & 0 & \ldots \\
0 & 1 & 0 & \cdots \\
0 & 0 & 0 & \cdots \\
0 & 0 & 1 & \cdots \\
\cdots & \ldots & \ldots
\end{array}\right\|,
\end{aligned}
$$

so that $T[c] \subset c_{0}$ and $T U=I$. 
Then $T$ is not a right topological divisor of 0 in $\Gamma$ since for any matrix $S \in \Gamma,\|S\|=\|S T U\| \leqq\|S T\| \cdot\|U\|$. But $T$ is even a right divisor of 0 in $B[c]$, since $S T=0$ if $S=1 \otimes \mathrm{lim}$.

However, if we call a right topological divisor of zero proper whenever it is not a right divisor of zero, then the result corresponding to Lemma 11 is correct.

LEMMA 12. The first result of Lemma 11 is correct with "left" replaced by "proper right topological."

The fact that $T$ is a matrix will not be used.

We begin the proof with a technical lemma which is essentially Lemma 1 of [2].

LEMMA 13. Let $X$ be a Banach space, and $E, F$ complementary closed subspaces such that $F$ is finite dimensional. If $T \in B[X]$ is one-to-one, and if $T: E \rightarrow X$ is a linear homeomorphism into, then $T: X \rightarrow X$ is a linear homeomorphism into.

We have $T[X]=T[E]+T[F] ; T[E]$ is closed by hypothesis, and $T[F]$ is finite dimensional. Hence $T[X]$ is closed. (See, for example, $[18$, Chapter $10, \S 6$ Corollary 5].) Since $T$ is also one-to-one, the result follows by the closed graph theorem.

To prove Lemma 12, we observe that $T$ has dense range, but is not onto, by [22, Theorems 3.3 and 3.6]. Hence, by [1, Theorem 1, p. 146], $T^{*}: c^{\prime} \rightarrow c^{\prime}$ is not a linear homeomorphism into; however, it is one-to-one since $T$ has dense range. It follows from Lemma 13 that $T^{*}: \Psi^{\perp} \rightarrow c^{\prime}$ is not a linear homeomorphism into; hence, for any $\varepsilon>0$, there exists $f \in \Psi^{\perp}$ with $\|f\|=1,\left\|T^{*} f\right\|<\varepsilon$. Let $U=1 \otimes f$. Then $\|U\|=1, U T x=f(T x) 1=T^{*} f(x) 1$ so that $\|U T\|<\varepsilon$, and $U$ is a matrix by Lemma $1(\mathrm{~d})$ since $f \in \Psi^{\perp}$.

We now have completed the adaptation of Ycod's results [22] to the smaller algebra $\Gamma$.

Recall that, in the present context, "onto" and "one-to-one" mean "onto $c$ " and "one-to-one on c."

THEOREM 5. Let $T \in \Gamma$. Then

$T$ is a left divisor of 0 in $\Gamma$ if and only if it is not one-to-one.

$T$ is a left topological divisor of 0 in $\Gamma$ if and only if it is not a linear homeomorphism into, i.e., either $T$ is not one-to-one or $T$ is not range-closed.

$T$ is a proper right topological divisor of 0 in $\Gamma$ if and only if it has dense range and is not onto.

Let $T$ be coregular. Then $T$ is a right divisor of 0 in $\Gamma$ (i.e., is not of type $M)$ if and only if the range of $T$ is not dense.

This follows from Lemmas 11, 12 and Corollary 2, with [22], Theorems 3.2, 3.5, Corollary 3.4 and Theorem 3.6, Theorem 3.3, respectively. 
In an unpublished thesis (Lehigh University, 1959) E. K. Dorff has obtained some other properties of $\Gamma$, for example, that it is a dense algebra of transformations of $c$. Some discussion of it is also given in [19].

\section{Summability.}

TheOREM 6 (I. D. Berg [2]). Suppose that $A \in \Gamma$ and that $A$ is not a left divisor of 0 . Then $A$ sums bounded divergent sequences if and only if $A$ is a left topological divisor of 0 in $\Gamma$.

Equivalently,

THEOREM 6'. Suppose that a conservative matrix $A$ is one-to-one on $c$. Then $A$ sums bounded divergent sequences if and only if to each $\varepsilon>0$ corresponds a conservative matrix $B$ with $\|B\|=1,\|A B\|<\varepsilon$.

Necessity follows from the fact [20, Theorem 1] that $c$ is not a closed subset of the $F K$ space $c_{A}$. Thus, given $\varepsilon>0$, we may find $x \in c$ with $\|x\|=1,\|A x\|<\varepsilon$, since $\|A x\|$ is one of the seminorms defining the $F K$ topology of $c_{A}$. Then $B=x \otimes P_{1}$ satisfies the condition stated. For a reversible matrix, this argument also yields the converse result. We refer to Berg's article for a complete proof. It may also be deduced from [6, Theorem 4].

COROLlaRY 3. Every conull matrix is a left topological divisor of 0 in $\Gamma$.

S. Mazur and W. Orlicz [12, p. 155, line 20] gave the first half (necessity) of the following result under the more restrictive hypothesis that $A$ is one to-one on $m$. For reversible matrices it is equivalent to [20, Theorem 1].

THEOREM 7. Let $A$ be a conservative matrix which is one-to-one on $c$. Then $A$ sums no bounded divergent sequence if and only if $A[c]$ is closed in $c$.

This follows from Theorems 5 and 6.

The following result is suggested by a theorem of Tropper and Martin. See the discussion of Theorem 10, below, for details.

THEOREM 8. Let $A$ be a conservative matrix which is one-to-one on $c$. Then $A$ sums bounded divergent sequences if and only if there exists a matrix $B$ with convergent columns such that $\|B\|=\infty$, but $\|A B\|<\infty$.

If $B$ exists, for $n=1,2, \cdots$, let $B_{n}$ be the matrix gotten from $B$ by replacing all terms not in the first $n$ columns by 0 . Then $B_{n} \in \Gamma,\left\|B_{n}\right\| \rightarrow \infty$, and $\left\|A B_{n}\right\|$ $\leqq\|A B\|<\infty$, so that $A$ is a left topological divisor of 0 . By Theorem $6 ; A$ sums bounded divergent sequences.

Conversely, assume that $A$ sums a bounded divergent sequence. By Theorem 6 and Lemma 8, it follows that given $\varepsilon>0$, we may find $x \in c$ with $\|x\|=1,\|A x\|<\varepsilon$. For $r=1,2, \cdots$, let $x^{r} \in c$ satisfy $\left\|x^{r}\right\|=r,\left\|A x^{r}\right\|<2^{-r}$. Let $B$ be the matrix whose $r$ th column is $x^{r}$. Then $\|B\|=\infty,\|A B\|<\Sigma 2^{-r}=1$. 
The first part of the following result is due to J. Copping [4]. See also [21, Theorem 1]. The second part was given by J. Copping [6, Theorem 4] under the more restrictive hypothesis that $A$ is one-to-one on $m$.

THEOREM 9. If a conservative matrix $A$ has a left inverse matrix $B$ with $\|B\|<\infty$, then $A$ sums no bounded divergent sequences and $A$ has a conservative left inverse.

If a conservative matrix $A$ is one-to-one on $c$, then $A$ sums no bounded divergent sequences if and only if $A$ has a conservative left inverse.

If $\|B\|<\infty$ and $B A=I$, it is clear that $A$ is not a left topological divisor of 0 . Hence, by Theorem $6, A$ sums no bounded divergent sequences. By Theorem 5 and Lemma 10, $A$ has a conservative left inverse. The last sentence of the statement follows in the same way from Theorem 6, Theorem 5 and Lemma 10.

It is worth remarking that if, in Theorem $9, A$ is permanent, the left inverse may be made permanent, and if $A$ is of type $M$ in addition, the left inverse must be permanent. This is proved in [6, p. 185]. (It would be trivial if $A$ were known to be onto.)

The next theorem is a considerable generalization of the result of A. M. Tropper [17], and C. F. Martin [11]. Their result referred to permanent triangular matrices with no zeros on the main diagonal, and is equivalent to the theorem given here in that special case.

THEOREM 10. Let $A$ be a conservative matrix which is one-to-one on $c$. Then $A$ sums bounded divergent sequences if and only if there exists a matrix $B$ with bounded columns such that $\|B\|=\infty$, but $\|A B\|<\infty$.

Suppose first that the matrix $B$ exists.

If $A$ sums no bounded divergent sequences, it has, by Theorem 9, a conservative left inverse $C$. Then $\|B\|=\|(C A) B\|=\|C(A B)\| \leqq\|C\| \cdot\|A B\|<\infty$, a contradiction. The associative law invoked in the second step applies because if $x$ is a column of $B, C(A x)=(C A) x$ by absolute convergence of a double series, since $x$ is bounded.

The converse follows from Theorem 8 .

Because of the depth of Theorem 9, it is worthwhile to sketch a more elementary proof of the first half (sufficiency) of Theorem 10.

We may assume that $A$ is one-to-one on $m$, for our hypothesis is that $A$ is oneto-one on $c$, and if $A x=0$ for some $x \in m \backslash c$ there is nothing to prove.

Just as in the proof of Theorem 8, the existence of $B$ implies that $A$ is a left topological divisor of 0 in the algebra of endomorphisms of $m$.

Let $\varepsilon>0$ be given. By Lemma 8 , there exists $x \in m$ with $\|x\|=1,\|A x\|<\varepsilon$. We can then find $\left\{\lambda_{n}\right\}$ with $\lambda_{n} \downarrow 0$ such that if $y_{n}=\lambda_{n} x_{n}$, we have $\|y\|=1,\|A y\|<\varepsilon$. (See for example [20, p. 503].) With Lemma 8 and Theorem 6, this completes the proof. 
6. Invariance of coregularity. J. Copping [5, Theorem 3] has deduced from a consistency theorem of Mazur-Orlicz-Brudno, the fact that "coregular" is an invariant of the bounded part of the convergence domain. Specifically, if $c_{A} \cap m$ $\subset c_{B} \cap m$ and $A$ is conull, so is $B$.

We should like to point out a different proof of this result.

Suppose that $A$ is conull and $c_{A} \cap m \subset c_{B}$.

Let $D=B-t I$ where $t=\chi(B)$. Then $D$ is conull, hence there exists a bounded divergent sequence $x$ which is summable by both $A$ and $D$ [23, Satz 3.4, II]. By hypothesis, it is summable by both $B$ and $D$. Thus $t x=B x-D x \in c$. Since $x \notin c$ it follows that $t=0$, i.e., $B$ is conull.

7. The boundary of the maximal group. In [6], and in improved form, in [2], a question raised in [19] was answered, concerning the boundary of the maximal group in $\Gamma$. Part of the question concerned the existence of a matrix summing bounded divergent sequences which is not on the boundary of the maximal group. An example is given in [6]. The following example involves less computation.

Let $A=\left(a_{n k}\right)$ where, for all $n, a_{n 1}=a_{n, 2 n}=1, a_{n k}=0$ otherwise. Then $A$ sums the sequence $\left\{(-1)^{n}\right\}$. Now $A[c]=c$ for, given $y \in c$ with $\lim y=t$, if we set $x=\left(t, y_{1}-t, 0, y_{2}-t, 0, y_{3}-t, 0, \cdots\right)$ we shall have $y=A x$. Thus, by Theorem $5, A$ is not a right topological divisor of 0 . (This could also be seen easily by noting that $A$ has a right inverse matrix of finite norm.) It follows that $A$ is not on the boundary of the maximal group. (A conull example is given in $\$ 9$, below.)

8. Steinhaus' theorem. The ideas of this article provide a setting for the classical result of Steinhaus that a permanent matrix cannot sum all bounded sequences, easily extended to coregular matrices by replacing $a_{n k}$ by $a_{n k}-\lim _{n \rightarrow \infty} a_{n k}$.

A matrix $A$ which maps $m$ into $c$ is called coercive;/such a matrix is conservative and indeed the coercive matrices are precisely the members of $\Gamma$ which are compact. (See, for example, [19, p. 381].) Each compact matrix is obviously the limit of a sequence of finite dimensional matrices. Any finite dimensional matrix is obviously conull, thus, since $\chi$ is continuous, every compact matrix is conull.This last statement is equivalent to Steinhaus' theorem. (It was pointed out in [3, p. 692], that no compact matrix can be permanent.)

\section{Questions.}

Note. A dittoed copy of this article was circulated pior to its publication with the result that questions I and IV have been answered by J. Lindenstrauss, questions IV and $\mathrm{V}$ by J. Copping, and V by R. J. Whitley.

I. Can $A \in \Gamma$ be conull and $A[c]=c$ ? 
Answer. Let

$$
A=\left\|\begin{array}{rrrrrrrrrr}
1 & 1 & -1 & 0 & 0 & 0 & 0 & 0 & . & . \\
1 & 0 & 0 & 1 & -1 & 0 & 0 & 0 & . & . \\
1 & 0 & 0 & 0 & 0 & 1 & -1 & 0 & . & . \\
. & . & . & . & . & . & . & . & . & . \\
. & . & . & . & . & . & . & . & . & .
\end{array}\right\| .
$$

This yields a conull $A \in \Gamma$ which is not a right topological divisor of 0 , hence is not on the boundary of the maximal group. Being conull, it sums bounded divergent sequences.

Dr. Berg pointed out in a conversation that the matrix of [21, p. 403] carries $c$ onto $c_{0}$, that no triangle may do this-by Theorem 3-and no triangular matrix may do it either since a triangular matrix map onto $c_{0}$ must be a triangle by easy induction on finite dimensional subspaces of $c_{0}$.

There is also no matrix projection of $c$ onto $c_{0}$ since $[16, \mathrm{p}$. 547] shows that the general projection of $c$ onto $c_{0}$ is $I-u \otimes \lim$, where $\lim u=1$.

II. Which of the classical summability methods, Norlund, Euler, Hausdorff, etc., are on the boundary of the maximal group? Mercer's theorem says that $(C, 1)$ is. My guess is that they all are.

III. The function $\chi$ is of no interest for nonmatrices. Possibly something better might be suggested, for example if we set $\rho(T)=\chi(T)-\lim _{n \rightarrow \infty} \chi_{n}(T)$, we shall have $\rho(T)=\chi(T)$ if $T$ is a matrix. Moreover $\rho(u \otimes f)=0$ for all $u, f$, a very reasonable situation. Unfortunately $\rho$ is not defined for all $T \in B[c]$. For example, if $t \in m \mid c$, define $T \in B[c]$ by $P_{n} \circ T x=t_{n}\left(\lim x-x_{n}\right)$. Then $\chi_{n}(T)=t_{n}$, so that $\rho(T)$ does not exist.

IV. I conjecture that if a conservative matrix $A$ sums a bounded divergent sequence, there cannot be a continuous projection from $c_{A} \cap m$ onto $c$. In view of [10], this would imply the well-known result of Agnew, Zeller, and Mazur-Orlicz that $c_{A} \cap m$ cannot be separable in $m$. (Answer. Yes! This result, by J. Lindenstrauss appeared in the American Mathematical Monthly in 70 (1963), 977-978. J. Copping also has a proof.)

V. I conjecture that if a conservative matrix $A$ sums bounded divergent sequences, the same is true of the matrix gotten from $A$ by multiplying various columns of $A$ by -1 . If $A$ is one-to-one on $c$ this follows from Theorem 9. For example the transformation $\left.x \rightarrow\left\{x_{n}+x_{n-1}\right)\right\}$ can be converted into the conull transformation $x \rightarrow\left\{(-1)^{n}\left(x_{n}-x_{n-1}\right)\right\}$ by this operation, hence sums bounded divergent sequences.

VI. Let $X=c$. Then $X$ has the property that $T \in B[X]$ is not a left topological divisor of 0 if and only if it has a left inverse (by Lemma 10 and Theorem 5). Which Banach spaces $X$ have this property?

A necessary and sufficient condition is, by [22, Theorem 3.15], that $X$ allows a bounded projection on each of its subspaces with which it is linearly homeo- 
morphic. The familiar condition $X=C[H]$ is sufficient, where $H$ is an extremally disconnected compact Hausdorff space, but is not necessary since $c$ is not of this form. Lemma 9 shows a technique for discussing the problem in the separable case, via projections from superspaces.

A sufficient, but not necessary, condition is that in the category of Banach spaces and bounded linear maps, the space $X$ is injective. For further information, see [9].

\section{REFERENCES}

1. S. Banach, Théorie des opérations linéaires, Monogr. Mat., Warsaw, 1932.

2. I. D. Berg, A Banach algebra criterion for Mercerian theorems, Proc. Amer. Math. Soc. (to appear).

3. L. W. Cohen and Nelson Dunford, Transformations on sequence spaces, Duke Math. J. 3 (1937), 689-701.

4. J. Copping, $K$-matrices which sum no bounded divergent sequence, J. London Math. Soc. 30 (1955), 123-127.

5. - Inclusion theorems for conservative summation methods, Nederl. Akad. Wetensch. Proc. Ser. 61 (1958), 485-499.

6. - Mercerian theorems and inverse transformations, Studia Math. 21 (1962), 177-194.

7. S. Hanai, On the methods of summation of infinite series, Tôhoku Math. J. 2 (1950), 64-67.

8. J. D. Hill, On perfect methods of summability, Duke Math. J. 3 (1937), 702-714.

9. Victor Klee, Relative extreme points, Review \#A2826, Math. Reviews 24 (1962), 526.

10. R. D. McWilliams, On projections of separable subspaces of $(m)$ onto $(c)$, Proc. Amer. Math. Soc. 10 (1959), 872-876.

11. C. F. Martin, A note on a recent result in summability theory, Proc. Amer. Math. Soc. 5 (1954), 863-865.

12. S. Mazur and W. Orlicz, On linear methods of summability, Studia Math. 14 (1954), 129-160.

13. M. S. Ramanujan, On summability methods of type $M$, J. London Math. Soc. 29 (1954), 184-189.

14. C. E. Rickart, Banach algebras, Van Nostrand, Princeton, N. J., 1960.

15. — The singular elements of a Banach algebra, Duke Math. J. 14 (1947), 1063-1077.

16. A. E. Taylor, The extension of linear transformations, Duke Math. J. 5 (1939), 538-547.

17. A. M. Tropper, A sufficient condition for a regular matrix to sum a bounded divergent sequence, Proc. Amer. Math. Soc. 4 (1953), 671-677.

18. A. Wilansky, Functional analysis, Blaisdell Press, New York, 1964.

19. A. Wilansky and K. Zeller, Banach algebra and summability, Illinois J. Math. 2 (1958), 378-385; ibid. 3 (1959), 468.

20. - Summation of bounded divergent sequences, topological methods, Trans. Amer. Math. Soc. 78 (1955), 501-509; ibid. 80 (1955), 386.

21. ——, The inverse matrix in summability: reversible matrices, J. London Math. Soc. 32 (1957), 397-408,

22. B. Yood, Transformations between Banach spaces in the uniform topology, Ann. of Math. (2) 50 (1949), 486-503.

23. K. Zeller, Faktorfolgen bei Limitierungsverfahren, Math. Z. 56 (1952), 134-151.

\section{LEHIGH UNIVERSITY,} Bethlehem, Pennsylvania 